$\underline{50}, 1564$ (1976).

${ }^{5}$ S. Nakahara, R. Schutz and L. R. Testardi, in Proceedings of the International Conference on Metallurgical Coatings, San Diego, 1980 (to be published).

${ }^{6}$ A. Segmüller and A. E. Blakeslee, J. Appl. Cryst. $\underline{6}$, 19 (1973).

${ }^{7}$ The films here will be specified by the ideal $\mathrm{Ni}-\mathrm{Cu}$ thicknesses. However, this is not meant to imply that sharp boundaries were achieved in the films.

${ }^{8}$ I. S. Jacobs and C. P. Bean, in Magnetism III, edited by G. Rado and H. Suhl (Academic, New York, 1963), p. 276. G. P. Felcher, J. W. Cable, J. Q. Zheng, J. B. Ketterson and J. E. Hilliard (private communication) have recently discussed clustering in these materials.

${ }^{9}$ R. M. White and C. Herring, to be published.

${ }^{10}$ U. Gradman, J. Magn. Magn. Mater. $\underline{6}, 173$ (1977).

\title{
Theory for the Dielectric Function of Granular Composite Media
}

\author{
Ping Sheng \\ Theoretical Sciences Group, Exxon Research and Engineering Company, \\ Corporate Research Science Laboratories, Linden, New Jersey 07036
}

(Received 28 March 1980)

\begin{abstract}
A new theory for the dielectric function of composite solids is formulated which displays both the optical dielectric anomaly and the percolation threshold, thereby providing a basis for a unified understanding of the optical and percolation transport properties of granular materials. The results of the theory are shown to be in good agreement with experimental data.
\end{abstract}

PACS numbers: $77.90 .+\mathrm{k}$

Recent interest in the optical and transport properties of granular composite materials has spurred renewed theoretical investigation in the calculation of the dielectric function ${ }^{1-3}$ for a heterogeneous composite medium. There are at present two prevalent theories for the dielectric constant of composite materials. One is the Maxwell-Garnett theory ${ }^{3}$ (MGT), which is usually preferred for the calculation of optical properties, because it predicts the existence of the optical dielectric anomaly observed in granular metal films. However, because of the inherently asymmetrical treatment of the two constituents of the composite, MGT predictions grossly disagree with experimental optical and transport results in cermets when the volume fraction of the dispersed phase (in MGT) becomes comparable to or greater than that of the matrix phase. ${ }^{3}$ In particular, the theory does not produce the observed percolation threshold in granular metals. The effective medium theory (EMT) proposed by Bruggeman $^{4}$ is the other widely used approach to the calculation of the dielectric constant for composite materials. The EMT does give a percolation threshold, but, unlike the MGT, it yields no dielectric anomaly. Moreover, the predicted value of the percolation threshold is low compared with the experimental result. ${ }^{5}$

In this Letter I present a new theory for the dielectric constant of granular composite (also known as cermet films). The theory displays both the optical dielectric anomaly and the percolation threshold, thereby providing a basis for the unified understanding of the optical and percolation transport properties of granular composite media.

The study of hopping conductivity in grangular metals ${ }^{6-8}$ has indicated that the microstructure of composite films is primarily determined by the grain formation process through surface diffusion of sputtered or evaporated molecules. The resulting composition homogeneity on the scale of the surface diffusion length has been shown to be responsible for the characteristic temperature and electric field dependence ${ }^{8}$ of granular metal hopping conductivity. As the starting point of the present theory, consider a spherical region with the dimension of a diffusion length inside the material. Within such a region a fraction $p$ of the volume is taken by the molecules of component 1 and the rest by component 2 . Here $p$ is the macroscopic volume composition of component 1 . When a grain is formed inside this region by diffusion and coalescence. there are two possible outcomes: Component 1 forms the grain and component 2 the coating, which we denote as a type-1 unit; or component 2 may form the grain and component 1 the coating, which is denoted as a type-2 unit. The relative probability of occurrence for the two cases can be estimated by counting the number of equally possible final configurations (corresponding to 
different positions of the grain inside the region). By assuming the grain to be spherical, it is clear that, in the case of type 1 , the number of configurations is proportional to $v_{1}=\left(1-p^{1 / 3}\right)^{3}$, the free volume (up to a constant multiplicative factor) accessible by the grain center of mass inside the spherical region. By the same reasoning the number of configurations for the alternative case is proportional to $v_{2}=\left[1-(1-p)^{1 / 3}\right]^{3}$. It follows that, at any value of $p$, the relative probability of occurrence for type 1 unit is $f=v_{1} /\left(v_{1}+v_{2}\right)$, and that for type 2 unit is $1-f$. This simplified picture, though it neglects the interaction between neighboring regions in the grain-forming process, nevertheless does capture the essential structural variation in granular composites as a function of composition $p$. For $0 \leqslant p \leqslant 0.35$, the value of $f$ decreases slowly from 1 at $p=0$ to $\sim 0.92$ at $p$ $=0.35$. The preponderance of type- 1 unit in this range is in accord with the observed structure of grains of component 1 embedded in the matrix of component 2. As $p$ is increased from 0.35 to 0.65 , $f$ drops sharply, reaching $\sim 0.08$ at $p=0.65$. This composition region, with the two kinds of structural units competing for dominance, clearly corresponds to the structural transition regime seen in electron micrographs ${ }^{7}$ at similar values of $p$.
Beyond $p=0.65$, the matrix inversion is basically complete, and we have grains of component 2 dis persed in the matrix of component 1.

To calculate the dielectric constant of the composite, let us consider the embedding of a type-1 or a type-2 unit in a uniform effective medium of dielectric constant $\bar{\epsilon}$. Upon application of uniform electric field, the inclusion will produce a dipole moment $D_{1,2}(i)$, where the subscript corresponds to the type of unit and $i$ denotes the configuration. If we approximate $D_{1,2}(i)$ with the dipole moment of the concentric configuration, $D_{1,2}$, the effective-medium condition of zero average dipole moment ${ }^{9}$ results in the equation

$$
f D_{1}+(1-f) D_{2}=0
$$

It may be noted that Eq. (1) reduces to the MGT if $f$ is set equal to 1 . Although the discussion to this point has been confined to the case of spherical units, Eq. (1) is generally applicable to particles of spheroidal shapes as well, since the preceding arguments remain unchanged if one considers a spheroidal particle enclosed in a similar-shaped region. In that general case, however, $D_{1,2}$ stands for the orientationally averaged dipole moment of coated confocal spheroidal particles embedded in an effective medium $\overline{\boldsymbol{\epsilon}}$ :

$$
\begin{aligned}
& D_{1}=\frac{2}{3} D\left(\bar{\epsilon}, \epsilon_{1}, \epsilon_{2}, p, A(\alpha, u), B(\alpha)\right)+\frac{1}{3} D\left(\bar{\epsilon}, \epsilon_{1}, \epsilon_{2}, p, 3-2 A(\alpha, u), 3-2 B(\alpha)\right), \\
& D_{2}=\frac{2}{3} D\left(\bar{\epsilon}, \epsilon_{2}, \epsilon_{1}, 1-p, A(\beta, v), B(\beta)\right)+\frac{1}{3} D\left(\bar{\epsilon}, \epsilon_{2}, \epsilon_{1}, 1-p, 3-2 A(\beta, v), 3-2 B(\beta)\right),
\end{aligned}
$$

where $\alpha$ is the ratio between the minor (major) and major (minor) axes of the elliptic cross section for the type-1 oblate (prolate) spheroidal unit, $\beta$ is the similar quantity for the type-2 unit, $u=(p / \alpha)^{1 / 3}$, and $v=[(1-p) / \beta]^{1 / 3} . D, A$, and $B$ have the following functional forms:

$$
\begin{aligned}
& D(\bar{\epsilon}, x, y, \mu, A, B)=\frac{[A \bar{\epsilon}+(3-A) y](y-x) \mu+[B x+(3-B) y](\bar{\epsilon}-y)}{A(3-A)(\bar{\epsilon}-y)(y-x) \mu+[B x+(3-B) y][A y+(3-A) \bar{\epsilon}]}, \\
& A(\gamma, w)=\frac{3}{2} \frac{1}{\left(1-\gamma^{2}\right) w^{3}}\left[\frac{1}{\left(1-\gamma^{2}\right)^{1 / 2}} \tan ^{-1} \frac{\left(1-\gamma^{2}\right)^{1 / 2} w}{\left(s^{2}+\gamma^{2} w^{2}\right)^{1 / 2}}-w\left(s^{2}+\gamma^{2} w^{2}\right)\right],
\end{aligned}
$$

$B(\gamma)=A\left(\gamma, 1 / \gamma^{1 / 3}\right)$ evaluated at $s=0$, where $s$ is the solution of the equation $\left(s^{2}+w^{2}\right)^{2}\left(s^{2}+\gamma^{2} w^{2}\right)=1$, and $\tan ^{-1} i z \rightarrow i \tanh ^{-1} z$ where $\gamma>1$.

Equations (1)-(3) constitute a complete set of conditions for the determination of $\bar{\epsilon}$ from the input parameters $\epsilon_{1}, \epsilon_{2}, p, \alpha$, and $\beta$. To compare the theory with experimental results, we show in Fig. 1 the variation of normalized dc conductivity $\bar{\sigma}$ as a function of $p$ for $\mathrm{W}-\mathrm{Al}_{2} \mathrm{O}_{3}$ composite films. ${ }^{8}$ The solid lines are calculated with $\epsilon_{1}=i, \epsilon_{2}=0$, and the $\beta$ values indicated in the figure. $\alpha$ is assumed to be 1 for all systems in this paper because electron micrographs ${ }^{7}$ have shown the met- allic particles to be roughly round. Two features of Fig. 1 should be especially noted. First, it is seen that the annealed sample and the as-prepared sample exhibit opposite curvatures in the $\bar{\sigma}(p)$ variation. This characteristic trait is remarkably reproduced by the theory with two different values of $\beta$. The good agreement between theory and experiment therefore suggests that annealing modifies the geometry of the insulator particles from platelike before annealing to more spherical after annealing. The resulting increase in $\bar{\sigma}(p)$ upon annealing can then be simply explained 


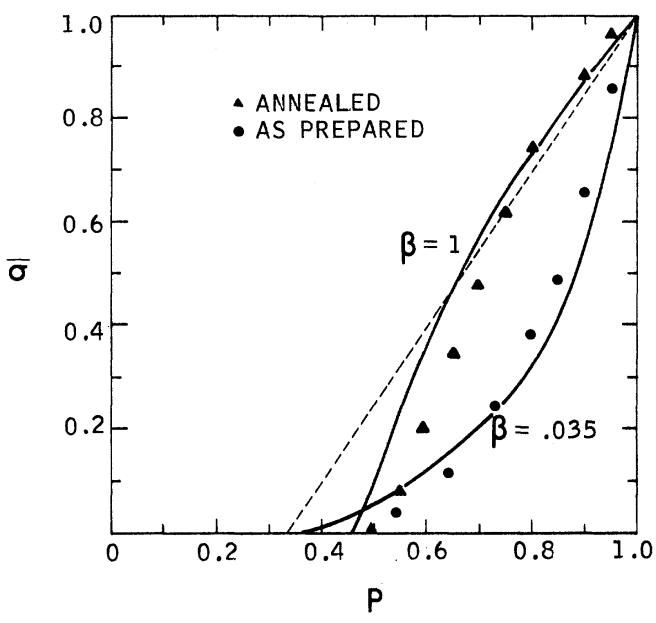

FIG. 1. Normalized conductivity $\bar{\sigma}$ plotted as a function of metal volume fraction $p$ for samples of $\mathrm{W}-\mathrm{Al}_{2} \mathrm{O}_{3}$ cermets. The data are from Ref. 7. Solid lines are calculated from the theory. Dashed line denotes EMT result.

by the fact that, for the same amount of insulator, platelets are expected to be more effective than spheres in impeding current flow. The second feature of the figure is the generally high value of percolation threshold compared with predictions of EMT and continuous random-percolation model. ${ }^{10}$ The result of the present theory shows that the structural constraint imposed by the condition of local composition homogeneity is sufficient to raise the percolation threshold to the observed range of $p_{c}=0.35-0.5$.

Optical transmission data of $\mathrm{Au}-\mathrm{SiO}_{2}$ composite films ${ }^{3}$ are plotted in Fig. 2 for five different $\mathrm{Au}$ compositions. ${ }^{11}$ The absorption peaks seen in these curves are the well-known dielectric anomalies peculiar to the optics of composite media. To obtain the theoretical curves, I have used for $\epsilon_{1}$ the experimentally known constants ${ }^{12}$ of $\mathrm{Au}$, modified to take into account the decrease of conduction electron relaxation time $\tau$ due to smallparticle microstructure. ${ }^{13}$ The value of $\tau$ used in the calculation is $2.5 \times 10^{-15} \mathrm{sec}$, corresponding to a particle size of $50 \AA$. Also, $\epsilon_{2}=2.2$ and $\beta=2$ were employed, where the value of $\beta$ is chosen by cursory survey of insulator particle shapes from electron micrographs. ${ }^{3}$ Since the absolute accuracy of experimental composition determination is $\sim \pm 5 \%$, the theoretical value of $p$ (labeled to the right of each curve) is treated as a slightly adjustable parameter in the calculation. Once $\bar{\epsilon}$ is known, the transmission through the film is

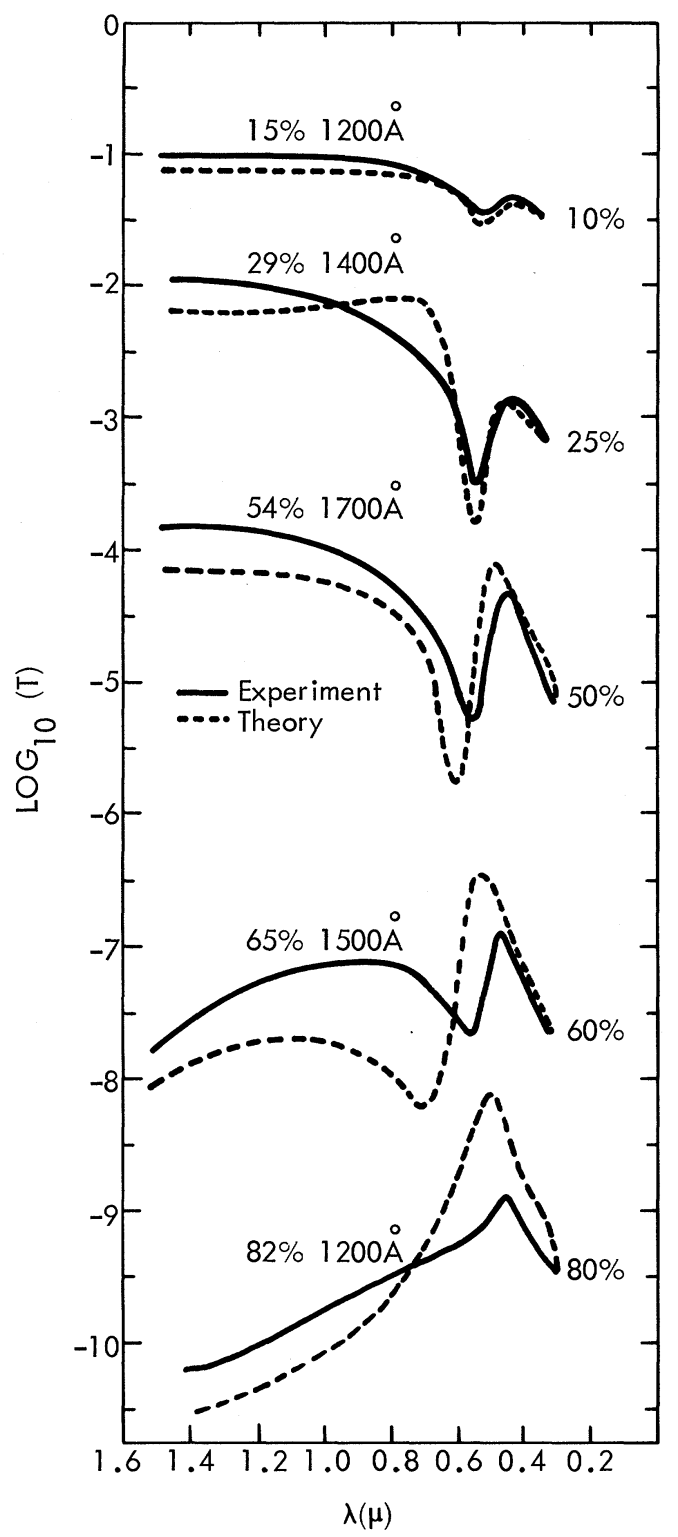

FIG. 2. Optical transmission as a function of light wavelength for a series of $\mathrm{Au}-\mathrm{SiO}_{2}$ composites. Data are from Ref. 3. For clarity, the curves are displaced with respect to one another. The theoretical curves are normalized to the experimental values at $0.3 \mu \mathrm{m}$. The theoretical values of $p$ are labeled to the right of pairs of curves, whereas the experimental values of $p$ and the film thickness are given above each pair of curves.

obtained by the usual electromagnetic wave formalism, ${ }^{14}$ with the experimentally determined film thickness values marked above each curve. From Fig. 2 it is noticed that the present theory reproduces all the characteristic features of the 


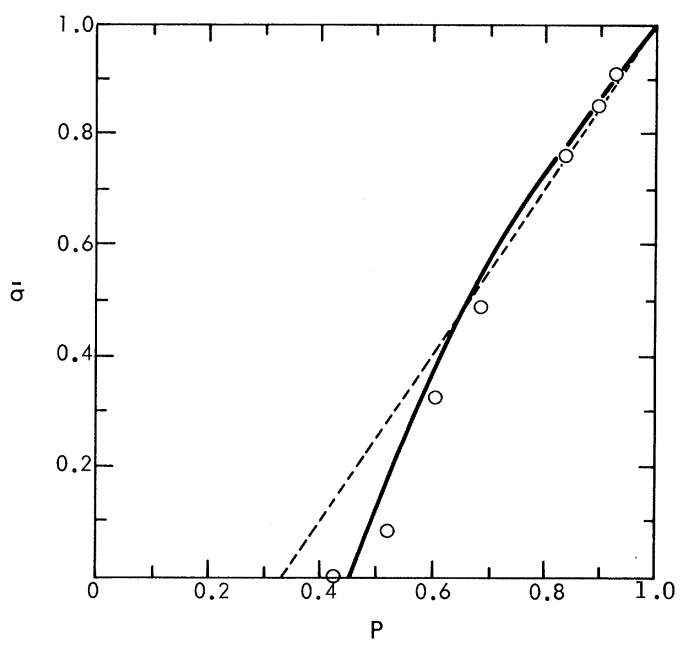

FIG. 3. Normalized conductivity $\bar{\sigma}$ as a function of $p$ for $\mathrm{Au}-\mathrm{SiO}_{2}$ cermets. Data from Ref. 3. Dashed line denotes EMT result.

data. Compared to MGT, the agreement of this theory with experimental results is far more satisfactory in two respects. First, whereas in the MGT the dielectric anomaly persists up to $p \simeq 1$, the new theory shows a rapid disappearance of the dielectric anomaly above $p=0.7$, in accord with observed data. Second, the MGT exhibits infrared behavior typical of the matrix component over the entire range of $p$. Therefore, if one (arbitrarily) chooses an insulator matrix, the infrared transmission will be independent of frequency for all $p$. However, in actual cases the infrared transmission undergoes a transition from being constant at small $p$ values to increasing with frequency at $p>0.6$. Such a transition is indeed correctly reproduced by the present theory. The dc electrical transport data ${ }^{3}$ of the Au$\mathrm{SiO}_{2}$ composites are displayed in Fig. 3. The solid line is calculated from the theory with the same value of $\beta$ as in Fig. 2. The excellent agreement with the experimental results confirms the self-consistency of the theory and demonstrates the essential underlying role of microstructure in both the optical and electrical properties of physical composite systems.

The author wishes to thank R. Cohen for helpful discussions and B. Abeles and R. Stephens for pointing out the revision in the values of cermet composition.

\footnotetext{
${ }^{1} \mathrm{~A}$ large number of articles and references may be found in Electrical Transport and Optical Properties of Inhomogeneous Media-1977, edited by J. C. Garland and D. B. Tanner, AIP Conference Proceedings No. 40 (American Institute of Physics, New York, 1978).

${ }^{2}$ D. Stroud and F. P. Pan, Phys. Rev. B 17, 1602 (1978).

${ }^{3}$ R. W. Cohen, G. D. Cody, M. D. Coutts, and B. Abeles, Phys. Rev. B 8 , 3689 (1973).

${ }^{4}$ D. A. G. Bruggeman, Ann. Phys. (Leipzig) 24, 636 (1935).

${ }^{5}$ B. Abeles, H. L. Pinch, and J. I. Gittleman, Phys. Rev. Lett. 35, 286 (1975).

${ }^{6}$ P. Sheng, B. Abeles, and Y. Arie, Phys. Rev. Lett. 31, 44 (1973).

${ }^{7}$ B. Abeles, P. Sheng, M. D. Coutts, and Y. Arie, Adv. Phys. 24, 407 (1975); B. Abeles, Appl. Solid State Sci. $\underline{6}, \overline{1}$ (1976).

${ }^{8}$ P. Sheng and B. Abeles, Phys. Rev. Lett. $\underline{28}, 34$ (1972).

${ }^{9}$ R. Landauer, J. Appl. Phys. 23, 779 (1952).

${ }^{10} \mathrm{H}$. Scher and R. Zallen, J. Chem. Phys. 53, 3759 (1970).

${ }^{11}$ The author has been advised by B. Abeles and R. Stephens that there is a systematic shift in the values of composition published in Ref. 4. The compositions used in the present paper represent the revised values.

${ }^{12}$ P. B. Johnson and R. W. Christy, Phys. Rev. B $\underline{6}$, 4370 (1972).

${ }^{13}$ See Ref. 3 for detailed prescription and justification of the procedure.

${ }^{14}$ O. S. Heavens, Optical Properties of Thin Solid Films (Dover, New York, 1965), p. 55.
} 